\title{
BMJ Open Impacts of heart disease, depression and their combination on all-cause mortality in older people: a rural community- based cohort study in China
}

\author{
Weiju Zhou (D) , ${ }^{1}$ Alex Hopkins, ${ }^{1}$ M Justin Zaman, ${ }^{2}$ Xuguang (Grant) Tao, ${ }^{3}$ \\ Amanda Rodney, ${ }^{1}$ Yuyou Yao, ${ }^{1,4}$ Zhongqiang Cao, ${ }^{5}$ Ying Ma, ${ }^{6,7}$ Zhi Hu, ${ }^{6}$ \\ John J Copeland, ${ }^{8}$ Ruoling Chen (i) ${ }^{1}$
}

To cite: Zhou W, Hopkins A, Zaman MJ, et al. Impacts of heart disease, depression and their combination on all-cause mortality in older people: a rural community-based cohort study in China. BMJ Open 2020;10:e038341. doi:10.1136/ bmjopen-2020-038341

- Prepublication history for this paper is available online. To view these files, please visit the journal online (http://dx.doi org/10.1136/bmjopen-2020038341).

Received 11 March 2020 Revised 18 October 2020 Accepted 23 October 2020

Check for updates

(C) Author(s) (or their employer(s)) 2020. Re-use permitted under CC BY-NC. No commercial re-use. See rights and permissions. Published by BMJ.

For numbered affiliations see end of article.

Correspondence to Professor Ruoling Chen; r.chen@wlv.ac.uk and Professor Zhi Hu; aywghz@126.com

\section{ABSTRACT}

Objective To assess the impact of heart disease (HD) combined with depression on all-cause mortality in older people living in the community.

Design A population-based cohort study.

Participants We examined the data of 1429 participants aged $\geq 60$ years recruited in rural areas in Anhui province, China. Using a standard method of interview, we documented all types of HD diagnosed by doctors and used the validated Geriatric Mental Status-Automated Geriatric Examination for Computer Assisted Taxonomy algorithm to diagnose any depression for each participant at baseline in 2003. The participants were followed up for 8 years to identify vital status.

Measurements We sought to examine all-cause mortality rates among participants with $\mathrm{HD}$ only, depression only and then their combination compared with those without these diseases using multivariate adjusted Cox regression models.

Results 385 deaths occurred in the cohort follow-up. Participants with baseline HD $(n=91)$ had a significantly higher mortality (64.9 per 1000 person-years) than those without HD (42.9). In comparison to those without HD and depression, multivariate adjusted HRs for mortality in the groups of participants who had HD only, depression only and both $\mathrm{HD}$ and depression were $1.46(95 \% \mathrm{Cl} 0.98$ to 2.17), $1.79(95 \% \mathrm{Cl} 1.28$ to 2.48$)$ and $2.59(95 \% \mathrm{Cl} 1.12$ to $5.98)$, respectively.

Conclusion Older people with both HD and depression in China had significantly increased all-cause mortality compared with those with HD or depression only, and without either condition. Psychological interventions should be taken into consideration for older people and those with $\mathrm{HD}$ living in the community to improve surviving outcome.

\section{INTRODUCTION}

Heart disease (HD) is the leading cause of death and accounts for about one-fifth of deaths worldwide. ' The term 'heart disease' refers to any disorders of the heart, and denotes various types of heart conditions including coronary heart disease (CHD),
Strengths and limitations of this study

- To the best of our knowledge, this study is the first to examine the impact of heart disease combined with depression on all-cause mortality in communitydwelling older people in low and middle-income countries.

- It included a wide range of well-characterised participants who lived in rural areas of China, being widely representative of low and middle-income lifestyles.

- The response rate of the participants was high both at baseline and follow-up of the cohort.

- There was a wider $\mathrm{Cl}$ for the estimated HR of mortality in patients with heart disease and concomitant depression due to the small number of patients who had both these conditions.

- The study has not examined the impacts of certain types of heart disease on all-cause mortality in older people.

hypertensive HD, cardiomyopathy and myocarditis, rheumatic HD, atrial fibrillation and flutter, or any other HDs. Previous studies have shown that depression is an independent risk factor for both incidence and mortality of $\mathrm{HD}^{2}{ }^{3}$ and people with $\mathrm{HD}$ are also more likely to suffer from depression. ${ }^{4}$ The relationship between HD and depression is bidirectional. ${ }^{5}$ HD and depression often coexist. $^{6}$

Previous studies in hospital settings showed that patients with HD and depression versus those without depression had increased mortality. ${ }^{7-9}$ Few studies have been undertaken in community settings to examine increased risk of mortality in older people with HD and depression compared with their counterparts who are free of HD and depression. ${ }^{1011}$ Older people have increased risks of HD and depression. ${ }^{12}$ The current knowledge of the association of $\mathrm{HD}$ or depression in older 
age with all-cause mortality is predominately derived from studies conducted in high-income countries (HICs). ${ }^{1} 1314$ The findings in HICs may not be generalisable to those in low and middle-income countries (LMICs). Importantly, despite the fact that the comorbidity of HD and depression increased in older age, little is known about the impact of HD combined with depression on all-cause mortality in older people, and no study has been undertaken in LMICs. Over the past three decades, HD mortality has fallen by more than half in HICs, but it has significantly increased in LMICs. ${ }^{15}$ In LMICs there are more and more people who have been affected by cardiac conditions as a result of the population ageing, epidemiological transition and risk factor changes, ${ }^{16}$ while rates of depression remain high. ${ }^{17}$ It is urgent to examine the impact of HD combined with depression on all-cause mortality in older communities in LMICs. China is the largest LMIC with 241 million older people. In this paper we examined the data of a community-based cohort study of older people in rural China, to assess the impacts of HD, depression and their combination on all-cause mortality.

\section{METHODS}

\section{Study population}

The participants were derived from the Anhui cohort study of older people's health in China. ${ }^{18}$ We examined the data of participants who lived in the rural areas, who were widely representative of a more typical LMIC lifestyle, away from the rapid modernisation of more urban populations. ${ }^{19}$ The methods of the baseline investigations ${ }^{19}$ and the follow-up in the rural Anhui cohort study ${ }^{18}$ have been fully described before. In brief, we randomly selected 1709 aged $\geq 60$ years from all 16 villages in Tangdian District of Yingshang county in 2003. A total of 1600 older people (754 men and 846 women) participated in the study, with a response rate of $93.6 \%$. Permission for interview and informed consent were obtained from each participant. In the case of those who were unable to provide informed consent due to disability or limited educational level, their next of kin or carer was invited to provide assent for participation. ${ }^{20}$ The participants were interviewed at home by a trained survey team from the School of Health Administration, Anhui Medical University, China. The main interview materials were a general health and risk factors questionnaire, and the Geriatric Mental Status (GMS) - a comprehensive semistructured mental state interview widely used in older people worldwide ${ }^{20}$ In the general health and risk factors record we documented details including: (1) sociodemographic information, (2) social networks and support, (3) psychological aspects, (4) doctor-diagnosed cardiovascular and other chronic disease, and (5) hobbies and activities of daily living. ${ }^{20}$ According to standard procedures ${ }^{18}$ we measured systolic and diastolic blood pressure, weight and height, and waist circumferences for all participants at baseline interview (wave 1 ).

\section{Baseline HD and depression}

At baseline interview, each participant (or their carers if the participant was unable to answer) was asked whether he or she had received a doctor-diagnosed type of any HD, with answer either 'Yes', 'No' or 'Unknown'. We performed a computer program-assisted diagnosis, the Automated Geriatric Examination for Computer Assisted Taxonomy (AGECAT), ${ }^{20}$ to assess the information from the GMS to identify the principal mental disorders in the study participants. The method of diagnosing depression has been described in previous publications. ${ }^{20}$ Briefly, AGECAT analyses of the GMS data bring symptom components together into groups that typify the major symptom areas of each diagnostic syndrome (organic disorder, depression, mania, schizophrenia and paranoia, obsessional, phobic, hypochondriacal and general anxiety). Individual participants were allocated to levels of confidence of diagnosis (0-5) on each of the eight diagnostic syndromes. The various syndrome levels were then compared with one another to derive a final differential diagnosis and a level of confidence of diagnosis from 0 to 5. A level of $\geq 3$, in most circumstances, designates a 'case level', which corresponds to a level of severity that warrants clinical intervention. Levels 1 and 2 are designated as 'subcases', while level 0 (no confidence level on any syndrome) is classified as 'well'. ${ }^{20}$ The GMS-AGECAT depression 'case' diagnoses have been compared with psychiatrists' diagnoses and Diagnostic and Statistical Manual of Mental Disorders, Third Edition criteria and applied with good levels of agreement in a variety of settings, including older people in China. ${ }^{21}$

\section{Follow-up of the cohort}

Vital status of the cohort members was monitored until December 2011. At each wave survey we conducted home visits to obtain information about participants' survival status through multiple sources including villagers' committees, family members, neighbours and friends. Using a standard verbal autopsy questionnaire we interviewed the next of kin responsible for the deceased or reviewed the death certificate to ascertain causes of death. ${ }^{20}$ We successfully followed up 1429 cohort members at wave 2, wave 3 or wave 4, with an overall rate of $89.3 \%$.

\section{Patient and public involvement}

We collected data from community-based dwelling older adults. Participants were not involved directly in the development of, recruitment to and conduct of the study. They did not have any role in study design, data analysis, data interpretation or writing of the manuscript.

\section{Statistical analysis}

Distributions of sociodemographic and risk factors between participants with and without HD were assessed by a t-test for continuous variables and a $\chi^{2}$ test for category variables. We employed multivariate Cox regression models to calculate HR and $95 \%$ CI of all-cause mortality among participants with HD versus those without. In 
the models we adjusted different sets of confounders, including depression to examine the association of HD with all-cause mortality. Then we investigated and compared mortality in patients who had HD only, depression only and both HD and depression in comparison to those without any HD or depression. The proportional hazards assumption in the Cox models was checked and satisfied for the covariates. All data were analysed using the SPSS statistical package (Windows V.24.0; SPSS).

\section{RESULTS}

Of 1429 participants, the average age was 70.9 years, and $51.5 \%$ were women. Their basic characteristics are illustrated in table 1. At baseline investigation, 91 participants were documented with HD. They were more likely to be obese, walk (or tour in group) and have diabetes, but less likely to drink alcohol in comparison to those without HD. They had a $50 \%$ increase in depression, which was not statistically significant. There were no significant differences in age, sex, smoking status, levels of education, social networks and support, hypertension, stroke and dementia between HD and non-HD participants (table 1).

Over 8 years follow-up of the cohort, 385 participants died. The mortality was higher in participants with HD (64.87 per 1000 person-years) than those without HD (42.85 per 1000 person-years) $(\mathrm{p}=0.021)$. Age-sex adjusted HR for mortality in participants with HD was $1.52(95 \%$ CI 1.07 to 2.16), and with further adjustment for body mass index (BMI), smoke, alcohol drinking, educational level, living status, frequency of visiting children or relatives, and walking or group touring, the HR was $1.48(95 \%$ CI 1.04 to 2.12). This was not substantially changed after adding comorbidities for adjustment, including depression (table 2).

Table 3 shows mortality and adjusted HRs among the four groups of participants in terms of HD and depression conditions. There were significant differences in mortality among these groups $(p=0.011): 41.00$ per 1000 person-years in participants with neither HD nor depression, 62.65 per 1000 person-years in HD only, 60.46 per 1000 person-years in depression only and 77.72 per 1000 person-years in participants with both HD and depression. The age-sex adjusted HR for mortality in participants with HD only, with depression only and with both HD and depression in comparison to those without HD and depression was 1.52 (95\% CI 1.03 to 2.24), 1.63 (95\% CI 1.20 to 2.22 ) and 2.17 (95\% CI 0.96 to 4.91 ), respectively. Further adjusted for other variables including BMI, smoke, alcohol drinking, educational level, living status, frequency of visiting children or relatives, walking or group touring, hypertension, diabetes and dementia, the matched HRs for mortality in the groups of participants who had HD only, depression only and both were 1.46 (95\% CI 0.98 to 2.17 ), 1.79 (95\% CI 1.28 to 2.48 ) and 2.59 (95\% CI 1.12 to 5.98 ), respectively (table 3 ).

\section{DISCUSSION}

Our cohort study from China demonstrated that older people with HD had a $46 \%$ increase in all-cause mortality compared with those without HD. Further data analysis for the participants with both HD and depression showed increased mortality at $159 \%$ while mortality in patients with HD only was increased at $46 \%$, and with depression only at $79 \%$, compared with those without HD and depression.

Current literature shows that few studies have examined the impact of HD on all-cause mortality in the communitydwelling older people, particularly in LMICs. In our study of China, data of HD, no matter with or without depression, revealed a $46 \%$ increase in all-cause mortality. The finding is consistent with an Australian study, which included a community-derived sample of 4805 participants aged 69-87 with a mean follow-up of 6.0 years and showed that the risk of all-cause mortality was increased in those with CHD (HR $1.6,95 \%$ CI 1.4 to 1.8$).{ }^{22}$

Our study also demonstrated that older people with depression in China have increased all-cause mortality, which was not confounded by other factors including HD and other cardiovascular risk factors. A recent systematic literature review and meta-analysis, which included 49 cohort studies, found that late-life depression increased the risk of all-cause mortality by $34 \% .{ }^{14}$ The figure was mainly derived from studies undertaken in HICs. In comparison, our study showed a stronger impact of depression on all-cause mortality. The HR in patients with depression combined with HD was 2.59, showing a multiplicative production of HRs of depression and HD. These infer the need for more detection and treatment of depression in older people in rural China, particularly in those with $\mathrm{HD} .{ }^{23}$ In rural China, older people had a low level of using mental health services, ${ }^{2425}$ and there is lack of trained medical staff who can identify, diagnose and treat psychiatric conditions, ${ }^{26}$ contributing further to consequent under-recognition and undertreatment. This may be one of the reasons for the stronger impact of late-life depression on increased allcause mortality in rural China.

Depression is highly prevalent among patients with HD in China ${ }^{27}$ and other countries. A study conducted in the urban community, China showed a $34.6 \%$ of depression in patients with CHD aged $>60$ years, ${ }^{28}$ while there was no study in the rural community. Our study showed a $15.4 \%$ of depression (case and subcase) in older people with HD in rural China. Although many studies in hospital settings showed that patients with HD after experiencing depression would have a worse prognosis and increased mortality, ${ }^{7-9}$ few studies have been done in the communitydwelling settings to examine the impact of HD combined with depression on all-cause mortality and only two studies to quantify the magnitude of mortality increased in people with HD and depression in comparison to those with neither. ${ }^{10}{ }^{11}$ The British Whitehall II study followed up 5936 participants aged 35-55 years for a mean of 5.6 years, ${ }^{10}$ and the age-sex adjusted analysis showed that HR for all-cause mortality was 1.67 (95\% CI 1.05 to 2.66) in 
Open access

Table 1 Characteristics of participants with and without heart disease at baseline in the Anhui rural cohort study, China

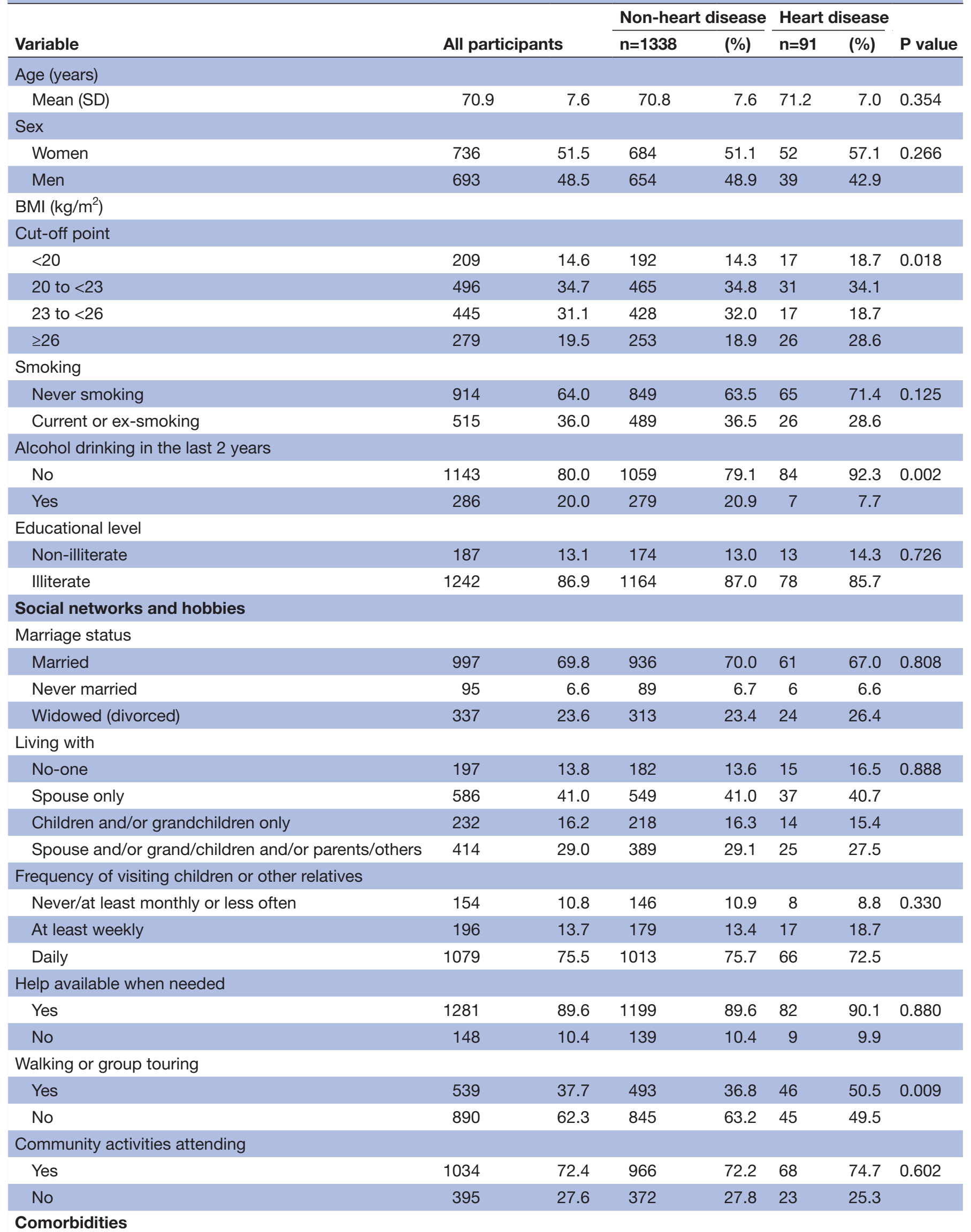


Table 1 Continued

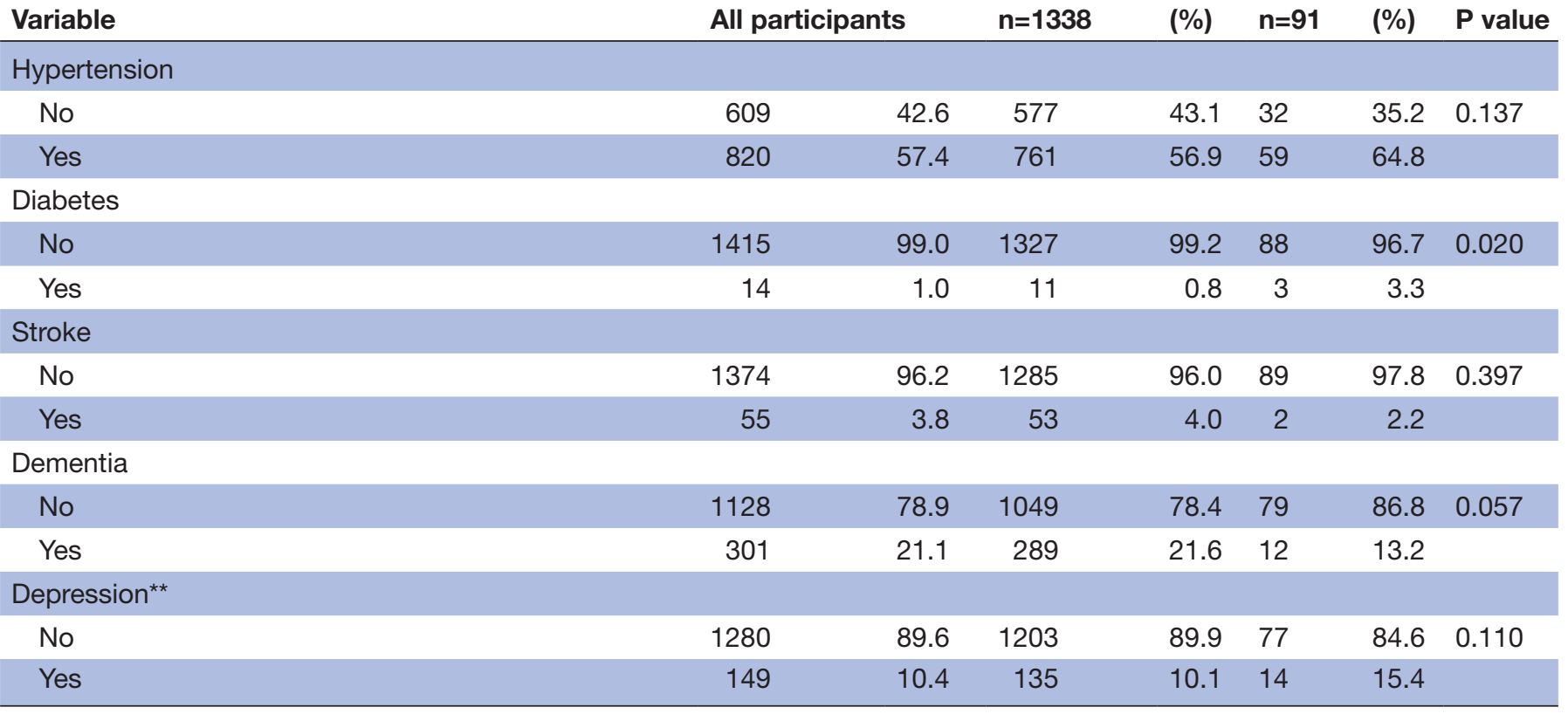

*In this paper, we included participants who had any levels of Geriatric Mental Status-Automated Geriatric Examination for Computer Assisted Taxonomy (GMS-AGECAT) depression as having depression.

$\mathrm{BMI}$, body mass index.

patients with CHD only, 2.10 (95\% CI 1.41 to 3.13) in depression only and 4.99 (95\% CI 2.89 to 8.62$)$ in having both CHD and depression in comparison to people with neither of them. ${ }^{10}$ However, after further adjustment for socioeconomic status, lifestyles and cardiovascular disease risk factors, these HRs were substantially reduced: 1.10 (95\% CI 0.66 to 1.82 ) in patients with CHD, 1.78 (95\% CI 1.17 to 2.69) in depression and 2.94 (95\% CI 1.63 to 5.34) in CHD combined with depression. ${ }^{10}$ In the Netherlands, Penninx et al followed up a cohort of 2847 people aged 55-85 years for averaged 4.2 years and observed that compared to those without cardiac disease and depression, mortality was increased in patients with cardiac disease only (multivariate adjusted HR 3.4, 95\% CI 2.4 to 4.9) and in depression only (minor depression $1.6,95 \%$
CI 1.0 to 2.8; major depression $3.8,95 \%$ CI 1.4 to 10.6 ), while patients who had cardiac disease with depression had the highest risk of mortality (with minor depression $5.1,95 \%$ CI 3.1 to 8.6 ; with major depression 10.5 , $95 \%$ CI 4.1 to 26.7). ${ }^{11}$ Our study in China found that older people with HD and depression had a higher risk of all-cause mortality than those with either HD or depression alone. Our data from LMICs have contributed to the literature.

\section{Strengths and weaknesses of the study}

The main contribution of this study is to identify the combined effects of HD and depression on all-cause mortality in older people in rural communities of China. To the best of our knowledge, it is the first study to examine such effect in LMICs. Our study has limitations.

Table 2 Numbers of deaths and adjusted HR for all-cause mortality of heart disease among rural older adults in China: the Anhui cohort study

\begin{tabular}{|c|c|c|c|c|c|c|c|c|c|}
\hline $\begin{array}{l}\text { Heart } \\
\text { disease }\end{array}$ & $\begin{array}{l}\text { Deaths/participants, } \\
\text { n (\%) }\end{array}$ & $\begin{array}{l}\text { Person- } \\
\text { years }\end{array}$ & $\begin{array}{l}\text { Mortality } \\
\text { rate* }^{*}\end{array}$ & HR $^{1} 95 \% \mathrm{Cl}$ & $P$ value & $\mathrm{HR}^{2} 95 \% \mathrm{Cl}$ & $P$ value & $\mathrm{HR}^{3} 95 \% \mathrm{Cl}$ & $P$ value \\
\hline No & $351 / 1338(26.2)$ & 8191.6 & 42.85 & 1.00 & & 1.00 & & 1.00 & \\
\hline Yes & 34/91 (37.4) & 524.1 & 64.87 & $\begin{array}{l}1.52 \\
1.07 \text { to } 2.16\end{array}$ & 0.021 & $\begin{array}{l}1.48 \\
1.04 \text { to } 2.12\end{array}$ & 0.032 & $\begin{array}{l}1.46 \\
1.01 \text { to } 2.10\end{array}$ & 0.042 \\
\hline Total & $385 / 1429$ (26.9) & 8715.7 & 44.17 & & & & & & \\
\hline
\end{tabular}

$H R^{1}$ : adjusted for age (continuous), sex.

$\mathrm{HR}^{2}$ : adjusted for age (continuous), sex, body mass index (BMI), smoke, alcohol drinking, educational level, living status, frequency of visiting children or relatives, walking or group touring.

$\mathrm{HR}^{3}$ : adjusted for age (continuous), sex, BMI, smoke, alcohol drinking, educational level, living status, frequency of visiting children or relatives, walking or group touring, hypertension, diabetes, dementia and depression.

*Mortality rate per 1000 person-years. 


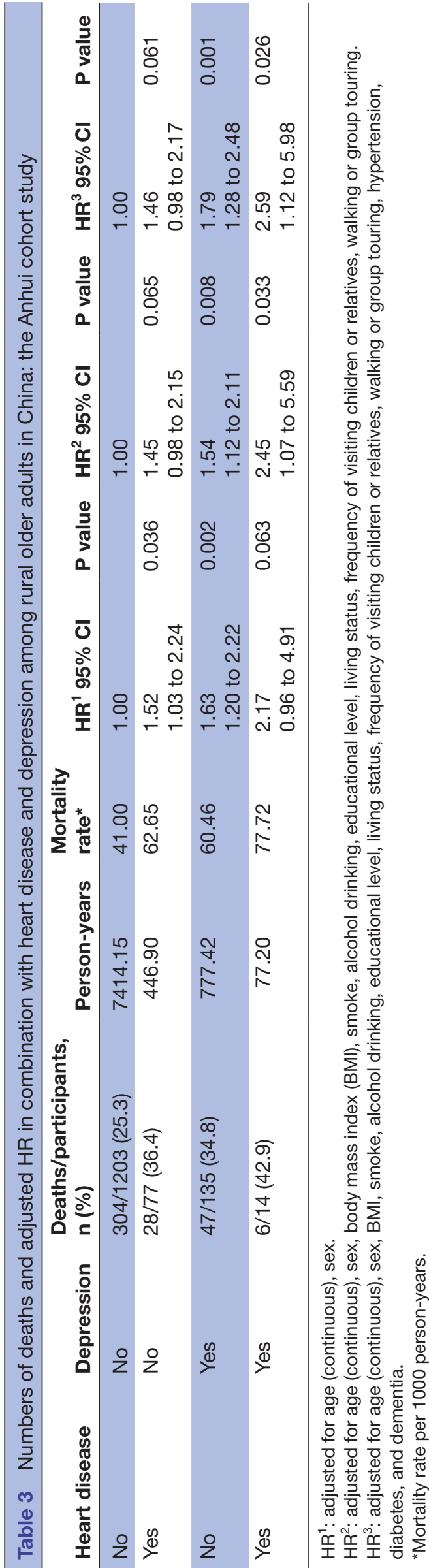

First, although our cohort had enough participants to be followed up for survival study, the number of patients who had both HD and depression was small, which made the HR for mortality a wider $95 \%$ CI. Second, our study included all types of HD, and we could not analyse data of a specific type of HD to investigate the impact of certain types of HD on mortality in older people. However, previous studies suggested that the effect of HD combined with depression on all-cause mortality is not sensitive to the definition of HD events. ${ }^{10}$ Third, the information of HD was collected using self-reported doctor diagnosis in the questionnaire, but not medical records. This may have caused some misclassification on HD diagnosis, biasing the direction of the association between HD and mortality towards null hypothesis. However, previous studies showed smaller differences between self-reported and physician reported in prevalence of HD in older adults. ${ }^{29}$ Our finding of using selfreported doctor-diagnosed HD is similar to that in the UK study, which used medical record. ${ }^{10}$ Nevertheless, a large cohort study of using hospital medical records is required to examine the association between HD and all-cause mortality in LMICs. Fourth, our study examined the impacts of HD and depression at baseline on all-cause mortality, not taking into account incident cases of HD and depression as well as depression recovering during the follow-up period. Although their numbers would be small, without including them in analysis, the associations of these health conditions with all-cause mortality analysed could have been underestimated, that is, the current findings in this paper would be more conservative. The impacts of HD, depression and their combination on all-cause mortality in older people need to be studied further in a large-scale cohort, with a shorter time interval (eg, 6 months) for reinterview to catch depression cases. Fifth, we acknowledge that the study is limited to the rural area in Anhui province, China, and its findings may not be generalisable to other rural areas in China. However, Anhui province is in the middle of China in terms of population density and economic status, and the findings of the study might still be applicable. Nevertheless, a larger scale cohort from multiple rural areas in China is likely to provide further confirmation of our findings.

\section{IMPLICATION AND CONCLUSION}

This study demonstrated that older Chinese with HD had an around $50 \%$ increase in all-cause mortality and there was additional effect of HD with depression on increased mortality. The findings highlight the importance of developing psychological interventions that are effective in reducing mortality among older people and those with HD who live in the rural communities in China. As the population of China continues ageing and the prevalence of HD and depression increase, it is vital to ensure that adequate mental health services and psychological support are available for vulnerable groups of older people. Early identification of depression in older people with and without HD, 
as well as appropriate prevention and treatment for depression in the populations, is a vital and attainable intervention to reduce mortality in China. Improving public health and mental healthcare in rural China will help achieve a healthier ageing society and attain better outcomes in older people with HD and depression.

\section{Author affiliations}

${ }^{1}$ Faculty of Education, Health and Wellbeing, University of Wolverhampton, Wolverhampton, UK

${ }^{2}$ Department of Cardiology, James Paget University Hospital, Norfolk, UK

${ }^{3}$ Department of Medicine, School of Medicine, Johns Hopkins University, Baltimore, Maryland, USA

${ }^{4}$ School of Public Health, Anhui Medical University, Hefei, Anhui, China

${ }^{5}$ Wuhan Children's Hospital (Wuhan Maternal and Child Healthcare Hospital), Tongji Medical College, Huazhong University of Science and Technology, Wuhan, Hubei, China

${ }^{6}$ School of Health Administration, Anhui Medical University, Hefei, Anhui, China ${ }^{7}$ Graduate College of Social Work, University of Houston, Houston, Texas, USA ${ }^{8}$ Institute of Psychology, Health and Society, University of Liverpool, Liverpool, UK

Acknowledgements The authors thank the participants and all who were involved in the rural Anhui cohort study.

Contributors RC (guarantor) obtained funding, conceptualised and designed the study, supervised the analyses and revised the manuscript. WZ performed the data analysis and drafted the initial manuscript. AH, MJZ, XGT, AR, YY, ZC, YM, ZH and JJC interpreted the findings, and commented and revised the manuscript. All authors approved the final version of the manuscript.

Funding The authors have not declared a specific grant for this research from any funding agency in the public, commercial or not-for-profit sectors.

Competing interests None declared.

Patient consent for publication Not required.

Ethics approval Ethical approval for the Anhui cohort study was obtained from the Ethics Committees of University College London, and School of Health and Wellbeing at University of Wolverhampton, UK, and the Research Ethics Committee of Anhui Medical University in China.

Provenance and peer review Not commissioned; externally peer reviewed. Data availability statement № additional data are available.

Open access This is an open access article distributed in accordance with the Creative Commons Attribution Non Commercial (CC BY-NC 4.0) license, which permits others to distribute, remix, adapt, build upon this work non-commercially, and license their derivative works on different terms, provided the original work is properly cited, appropriate credit is given, any changes made indicated, and the use is non-commercial. See: http://creativecommons.org/licenses/by-nc/4.0/.

\section{ORCID iDs}

Weiju Zhou http://orcid.org/0000-0001-5077-3202

Ruoling Chen http://orcid.org/0000-0002-3033-8753

\section{REFERENCES}

1 Lozano R, Naghavi M, Foreman K, et al. Global and regional mortality from 235 causes of death for 20 age groups in 1990 and 2010: a systematic analysis for the global burden of disease study 2010. Lancet 2012;380:2095-128.

2 Gan Y, Gong Y, Tong X, et al. Depression and the risk of coronary heart disease: a meta-analysis of prospective cohort studies. BMC Psychiatry 2014;14:371.

3 Carney RM, Freedland KE. Depression and coronary heart disease. Nat Rev Cardiol 2017;14:145-55.

4 Celano CM, Huffman JC. Depression and cardiac disease: a review. Cardiol Rev 2011;19:130-42.
5 Nemeroff CB, Goldschmidt-Clermont PJ. Heartache and heartbreak-the link between depression and cardiovascular disease. Nat Rev Cardiol 2012;9:526-39.

6 Rudisch B, Nemeroff CB. Epidemiology of comorbid coronary artery disease and depression. Biol Psychiatry 2003;54:227-40.

7 Barth J, Schumacher M, Herrmann-Lingen C. Depression as a risk factor for mortality in patients with coronary heart disease: a metaanalysis. Psychosom Med 2004;66:802-13.

8 Lichtman JH, Froelicher ES, Blumenthal JA, et al. Depression as a risk factor for poor prognosis among patients with acute coronary syndrome: systematic review and recommendations: a scientific statement from the American heart association. Circulation 2014;129:1350-69.

9 Gathright EC, Goldstein CM, Josephson RA, et al. Depression increases the risk of mortality in patients with heart failure: a metaanalysis. J Psychosom Res 2017;94:82-9.

$10 \mathrm{Nabi} \mathrm{H}$, Shipley MJ, Vahtera J, et al. Effects of depressive symptoms and coronary heart disease and their interactive associations on mortality in middle-aged adults: the Whitehall II cohort study. Heart 2010;96:1645-50.

11 Penninx BW, Beekman AT, Honig A, et al. Depression and cardiac mortality: results from a community-based longitudinal study. Arch Gen Psychiatry 2001:58:221-7.

12 Zhang Y, Chen Y, Ma L. Depression and cardiovascular disease in elderly: current understanding. J Clin Neurosci 2018;47:1-5.

13 Cuijpers P, Vogelzangs N, Twisk J, et al. Comprehensive meta-analysis of excess mortality in depression in the general community versus patients with specific illnesses. Am J Psychiatry 2014;171:453-62.

14 Wei J, Hou R, Zhang X, et al. The association of late-life depression with all-cause and cardiovascular mortality among communitydwelling older adults: systematic review and meta-analysis. $\mathrm{Br} \mathrm{J}$ Psychiatry 2019;215:449-55.

15 Finegold JA, Asaria P, Francis DP. Mortality from ischaemic heart disease by country, region, and age: statistics from World health organisation and United nations. Int J Cardiol 2013;168:934-45.

16 Gaziano TA, Bitton A, Anand S, et al. Growing epidemic of coronary heart disease in low- and middle-income countries. Curr Probl Cardiol 2010;35:72-115.

17 Ferrari AJ, Somerville AJ, Baxter AJ, et al. Global variation in the prevalence and incidence of major depressive disorder: a systematic review of the epidemiological literature. Psychol Med 2013;43:471-81.

18 Han TS, Wang HH-X, Wei L, et al. Impacts of undetected and inadequately treated hypertension on incident stroke in China. BMJ Open 2017;7:e016581.

19 Chen R, Wei L, Hu Z, et al. Depression in older people in rural China. Arch Intern Med 2005;165:2019-25.

20 Chen R, Hu Z, Wei L, et al. Socioeconomic status and survival among older adults with dementia and depression. $\mathrm{Br} J$ Psychiatry 2014;204:436-40.

21 Chen R, Hu Z, Wei L, et al. Severity of depression and risk for subsequent dementia: cohort studies in China and the UK. Br J Psychiatry 2008;193:373-7.

22 Almeida OP, Alfonso H, Flicker L, et al. Cardiovascular disease, depression and mortality: the health in men study. Am J Geriatr Psychiatry 2012;20:433-40.

23 Chen R, Hu Z, Chen R-L, et al. Determinants for undetected dementia and late-life depression. Br J Psychiatry 2013;203:203-8.

$24 \mathrm{Xu} \mathrm{J}$, Wang J, King M, et al. Rural-Urban disparities in the utilization of mental health inpatient services in China: the role of health insurance. Int J Health Econ Manag 2018;18:377-93.

25 Chen H-L, Xiao Y, Liu Y-J, et al. Treatment status of elderly patients with severe mental disorders in rural China. J Geriatr Psychiatry Neurol 2019;32:291-7.

26 Patel V, Xiao S, Chen $\mathrm{H}$, et al. The magnitude of and health system responses to the mental health treatment gap in adults in India and China. Lancet 2016;388:3074-84.

27 Ren $\mathrm{Y}$, Yang H, Browning C, et al. Prevalence of depression in coronary heart disease in China: a systematic review and metaanalysis. Chin Med J 2014;127:2991-8.

28 Yunming L, Changsheng $\mathrm{C}$, Haibo T, et al. Prevalence and risk factors for depression in older people in Xi'an China: a communitybased study. Int J Geriatr Psychiatry 2012;27:31-9.

29 Kehoe R, Wu SY, Leske MC, et al. Comparing self-reported and physician-reported medical history. Am J Epidemiol 1994;139:813-8. 